\title{
Pulsed intravenous methylprednisolone combined with oral steroids as a treatment for poorly responsive type 2 amiodarone-induced thyrotoxicosis
}

\author{
Irene Campi ${ }^{1,2}$, Giovanni B Perego ${ }^{3}$, Antonella Ravogli3 ${ }^{3}$, Antonella Groppelli ${ }^{3}$, Gianfranco Parati ${ }^{3,4}$, \\ Luca Persani ${ }^{1,5}$ and Laura Fugazzola ${ }^{1,2}$
}

1'Department of Endocrine and Metabolic Diseases, Istituto Auxologico Italiano, Istituto Di Ricovero e Cura a Carattere Scientifico (IRCCS), Milan, Italy, ${ }^{2}$ Department of Pathophysiology and Transplantation, University of Milan, Milan, Italy,

${ }^{3}$ Department of Cardiovascular Neural and Metabolic Sciences, Istituto Auxologico Italiano, Istituto Di Ricovero e Cura a Carattere Scientifico (IRCCS), San Luca Hospital, Milan, Italy, ${ }^{4}$ Department of Medicine and Surgery, University of Milan-Bicocca, Milan, Italy, and ${ }^{5}$ Department of Clinical Sciences and Community Health, University of Milan, Milan, Italy

Correspondence should be addressed to I Campi

Email

irene.campi@unimi.it

\begin{abstract}
Objectives: Amiodarone-induced thyrotoxicosis (AIT) affects up to 3\% of treated patients. Type 2 AIT (AIT2) is a destructive thyroiditis and is usually treated with medium-high oral doses of prednisone. As AIT may worsen the underlying heart disease, a rapid control of thyroid function is desirable. We aimed to determine whether a combined intravenous methylprednisolone (IVMP) pulses therapy associated to prednisone in the interpulse period can represent an efficient and safe alternative to urgent total thyroidectomy in patients with AIT2 not responsive to prednisone alone.

Design and methods: Patients presenting with a severe AIT2 studied in a tertiary referral Center from August 2018 to April 2019. We included four patients requiring a rapid improvement of thyroid function for their underlying cardiac disorders. The baseline doses of oral prednisone (range: 5-12.5 mg/day) and IVMP (range: 250-500 twice a week) were determined according to the severity of the thyrotoxicosis and were titrated based on clinical response.

Results: Combined treatment was effective in all patients in the prompt restoration of euthyroidism and no major adverse events were reported during the follow-up. In all cases, FT4 and FT3 levels normalized at 3-5 weeks of treatment. A permanent hypothyroidism was observed in one patient, 3 months after the discontinuation of treatment. Conclusions: We report for the first time that the combined intravenous and oral steroid therapy is effective in patients with AIT2. The treatment is well tolerated and leads to a rapid improvement of thyroid function, avoiding urgent total thyroidectomy and favoring a quick functional recovery and rehabilitation of cardiac patients.
\end{abstract}

\section{Introduction}

Amiodarone is an antiarrhythmic drug effective against a variety of cardiac arrhythmias, which may cause thyroid dysfunctions in nearly $30 \%$ of treated patients.

The incidence of amiodarone-induced thyrotoxicosis (AIT) compared to hypothyroidism are higher in iodine- (c) 2019 European Society of Endocrinology Printed in Great Britain deficient countries (1). Type 1 AIT (AIT1) occurs in patients with preexisting thyroid disease, while type 2 AIT (AIT2) is a destructive thyroiditis associated with release of preformed thyroid hormones (2). In mixed forms of AIT both mechanisms are present.

Published by Bioscientifica Ltd. 
AIT1 is usually responsive to thionamides and sodium/potassium perchlorate, while AIT2 is sensitive to oral prednisone. In mixed forms, the best strategy is the combination of these treatments. Independent of the etiology, it is advisable to get a rapid control of thyroid function, as AIT may worsen the underlying heart disease (2).

Although AIT2 is often considered as a mild selflimiting condition responsive to prednisone, it may present as a thyroid storm requiring urgent total thyroidectomy to rapidly restore euthyroidism, especially in patients with an ejection fraction lower than 30\% (3). Unfortunately, surgery is usually contraindicated in these patients, due to either the high anesthesiological risk or due to the lack of a dedicated multidisciplinary team (3). In selected cases, plasmapheresis may be considered, but it is relatively contraindicated in case of heart failure (4). The recommended first-line treatment for AIT2 is prednisone at the dose of $30 \mathrm{mg} /$ day titrated based on clinical response (3). Although poorly responsive patients, who require a high-dose long-term treatment often associated with adverse events exist, no therapeutic alternatives to prednisone have been proposed yet.

As the intravenous methylprednisolone (IVMP) administration allows reduction in steroid-related adverse events, we hypothesized that it might be an option in patients who need a prompt restoration of euthyroidism, allowing reduction of prednisone dose, increasing response rate and possibly preventing thyroidectomy.

Thus, we analyzed the efficacy and tolerability of combined IVMP pulses, associated with prednisone in the interpulse period in four patients with AIT2 diagnosed during hospitalization for cardiological diseases.

\section{Methods}

\section{Patients}

Four male patients were included in the study in the period July 2018-April 2019. All patients had been treated with amiodarone for 14-42 months (Table 1). Thereafter, the drug was withdrawn, in three cases at the diagnosis of thyrotoxicosis, and in patient \#2 due to bradycardia. In two patients (\#1 and \#3) the underlying cardiopathy was severe. All patients were initially treated with prednisone at standard doses $(37.5 \mathrm{mg} /$ day), associated or not with methimazole. They were all judged as not responsive, as FT4 levels were still above $40 \mathrm{pmol} / \mathrm{L}$ after at least 3 weeks of treatment (Table 1). In particular, patient \#1 was awaiting elective percutaneous coronary intervention (PCI) because of stenoses in the right anterior descending coronary and left circumflex artery (75 and 90\%, respectively) documented by cardiac CT scan. Patient \#3 was affected by chronic heart failure (ejection fraction $30 \%$ ), type 2 diabetes on insulin treatment, third-stage chronic kidney disease and peripheral artery disease (Table 1). AIT2 was diagnosed during routine preoperative tests for elective surgery of a $6 \mathrm{~cm}$ abdominal aortic aneurysm. Despite prednisone treatment, both patients had FT4 levels still >100 pmol/L, and patient \#3 underwent an episode of acute heart failure requiring hospitalization. In spite of increasing the dose of prednisone (up to $50 \mathrm{mg}$ in both), the FT4 levels did not improve; total thyroidectomy became the therapy of choice in these two patients, but the anesthesiologic and surgical risks were too high, and the IVMP treatment was started.

Patients \#2 and \#4 developed AIT2 8 months after amiodarone withdrawal and during amiodarone administration, respectively, and were both not responsive to prednisone treatment.

All patients gave their informed consent to the IVMP treatment and to the use of their clinical and biochemical data for scientific purposes. The study was approved by the Ethical Committee of Istituto Auxologico Italiano (Milan, Italy).

\section{Serum/urine assays and thyroid ultrasound}

Serum TSH, FT4, Tg, TPO-Ab, and Tg-Ab were measured on a Roche Cobas Eless 601 module immunology analyzer using the electrochemiluminescence immunoassay (ECLIA) method. Serum TSH-R antibodies (TRAbs) were measured as TSH-R binding inhibitory immunoglobulins by a third-generation TRAK human lumitest (ThermoFisher AG). 24-h urinary iodine excretion was measured by inductively coupled plasma mass spectrometry (ICP-MS).

Thyroid volume was measured by ultrasonography and calculated by the ellipsoid model. Body surface area (BSA)-corrected thyroid volume $\left(\mathrm{mL} / \mathrm{m}^{2}\right)$ was calculated according with the formula BSA $\left(\mathrm{m}^{2}\right)=\sqrt{\text { height }}$ $(\mathrm{m}) \times$ weight $(\mathrm{kg}) / 3600(5)$.

\section{Treatment schedule}

IVMP pulses were administered at 3-day interval and associated with prednisone during the intervals. The starting dose of each IVMP pulse was 250 or $500 \mathrm{mg}$, depending on the FT4 serum levels $(<4$ times the upper limit of normal value $=250,>4$ times the upper limit of normal value $=500$ ). Similarly, prednisone was administered at the initial dose of $5 \mathrm{mg} /$ day or 
Table 1 Clinical characteristic of patients included in the study.

Age
Gender
Duration of amiodarone treatment
(months)
Reason for discontinuation
Previous thyroid treatment for AIT before
combined therapy (weeks)
TSH (uUI/mL)
FT3 (pmol/L)
FT4 (pmol/L)
Tg-Ab (U/L)
TPO-Ab (U/L)
TRAb (U/L)
Thyroglobulin ( $\mu$ g/L)
$24-h$ urinary iodine excretion ( $\mu$ g/L/24 h)
99 mTc Thyroid uptake
Thyroid volume (mL)
BSA-corrected thyroid volume (mL/m²)
Steroid cumulative dose
(IVMP + oral prednisone mg)
Duration of the whole treatment with
steroid (weeks of IVMP administration)
Time to restore euthyroidism* (weeks)
Comorbidities

\begin{tabular}{|c|c|}
\hline Patient 1 & Patient 2 \\
\hline 66 & 61 \\
\hline Male & Male \\
\hline 25 & 14 \\
\hline AIT2 & Bradycardia \\
\hline Oral steroid (3) & $\begin{array}{l}\text { Methymazole (8), } \\
\text { oral steroid (4) }\end{array}$ \\
\hline$<0.005$ & $<0.005$ \\
\hline 17 & 12.8 \\
\hline$>100$ & 60.9 \\
\hline$<10$ & $<10$ \\
\hline$<9$ & $<9$ \\
\hline$<0.8$ & $<0.8$ \\
\hline 127 & 788 \\
\hline- & 2150 \\
\hline- & Absent \\
\hline 34 & 35 \\
\hline 15.4 & 19.7 \\
\hline $3250+1200$ & $3750+1140$ \\
\hline $10(5)$ & $17(6)$ \\
\hline 5 & 2 \\
\hline $\begin{array}{l}\text { Coronary disease, } \\
\text { IFG, hypertension, } \\
\text { obesity, AF }\end{array}$ & Hypertension \\
\hline
\end{tabular}

\begin{tabular}{l}
\hline Patient 3 \\
\hline 72 \\
Male \\
38
\end{tabular}

Patient 4

63

Male

42

AIT2

Oral steroid (3),

methymazole (12)

$<0.005$

10.9

$>100$

$<10$

$<9$

$<0.8$

433

1570

$-$

25

13.4

$1700+950$

AIT2

Methymazole (5), oral steroids (3) $<0.005$

8.7

41

$<10$

$<9$

$<0.8$

136

5300

$-$

13.4

6.6

$6500+580$

15 (9)

$14(11)$

3

3

Type 2 diabetes, AF, heart failure, abdominal aortic aneurysm, chronic kidney disease, peripheral artery disease

*Euthyroidism was defined as normal FT4 and FT3 levels even in the presence of a suppressed TSH.

AF, atrial fibrillation; BSA, body surface area; IFG, impaired fasting glycemia.

Normal reference rangess: TSH 0.3-4.5 uUI/mL; FT3 2.9-7.1 pmol/L; FT4 11.5-24.5 pmol/L; Tg-Ab < 115U/L; TPO-Ab <34 kU/L, TRAb < 1.5 U/L;

Thyroglobulin 0-85 $\mu \mathrm{g} / \mathrm{L} ; 24-\mathrm{h}$ urinary iodine excretion 100-200 $\mu \mathrm{g} / \mathrm{L} / 24 \mathrm{~h}, \mathrm{BSA}$-corrected thyroid volume: $<12 \mathrm{~mL} / \mathrm{m}^{2}$.

$12.5 \mathrm{mg} /$ day, depending on FT4 levels. Both steroids were tapered down based on FT4 reduction, and the decreasing rate of serum thyroglobulin (Tg) levels.

Once a stable euthyroidism (defined as normalization of both FT3 and FT4) was achieved, associated with constantly decreasing Tg levels, IVMP administration was stopped, while prednisone was continued (range 3-11 weeks). The initial daily prednisone dose was calculated as follows: $((1.25 \times$ IVMP $\mathrm{mg}) / 2) / 7,1 \mathrm{mg}$ of IVMP corresponding to $1.25 \mathrm{mg}$ of prednisone. Prednisone was progressively reduced every 10 days up to withdrawal, depending on biochemical response.

Since during destructive thyroiditis, the leakage of Tg occurs associated with an increase of its serum levels, we decided to use it as a marker. In particular, a decrease of Tg levels of at least $10 \%$ or its normalization suggested the reduction of the destructive process.

In order to prevent side effects of steroids treatment, we administered to all patients omeprazole, risedronate, calcium carbonate and colecalciferol during steroids treatment.

\section{Results}

At baseline, all patients had a severe thyrotoxicosis with FT4 levels ranging from 41 to $>100 \mathrm{pmol} / \mathrm{L}$ (normal values: $11.5-24.5 \mathrm{pmol} / \mathrm{L}$ ). The $24-\mathrm{h}$ urinary iodine excretion, measured in three out of four patients, was 7.5-26-fold higher than normal values (Table 1). In all patients TPO-Ab, Tg-Ab and TRAb were negative. Three patients had a simple goiter (thyroid volume range: $25-35 \mathrm{~mL}$ and $\mathrm{BSA}$-corrected thyroid volume range: 13.4-19.7), while one patient had a normal-sized thyroid. In patient \#2, who developed AIT several months after amiodarone discontinuation, a $99 \mathrm{mTc}$ thyroid scintigraphy was performed documenting the absence of uptake, consistently with the diagnosis (Table 1 ). The mean cumulative dose of IVMP and prednisone was $3.9 \mathrm{~g}$ (range: 1.7-7) and $0.968 \mathrm{~g}$ (range: 0.6-1.2) respectively (Table 1). Overall, the mean duration of corticosteroid treatment was 8 weeks (range: 5-14) (Table 1). FT4 and FT3 normalized during the first 3 and 5 weeks of treatment 
in three and one patients, respectively. Mean Tg levels declined over time along with the FT4 and FT3 levels (Fig. 1A and B and Fig. $2 \mathrm{~A}$ and B). Serum Tg levels were used as a parameter to decide the timing of steroid dose increase, as their rise anticipated of 1 week that of FT4. On the contrary, remission of hyperthyroidism was predicted by the constant decline of $\mathrm{Tg}$ levels regardless of the steroids reduction (Fig. 1A and B and Fig. 2 A and B).

During treatment, general biochemistry remained within the normal range except for impaired fasting glycemia in patients \#1 and \#4 (range 5.83-6.72 mmol/L) and a mild increase of gamma-glutamyltransferase in patient \#2 (highest value 88 UI/L; normal range 0-60). As expected, patient \# 3 required insulin dose adjustment during treatment.

In patient \#1 the improvement of thyroid function, with FT3 and FT4 in the normal range and progressively decreasing $\mathrm{Tg}$, allowed to safely perform a coronary angioplasty with stenting of the critical stenosis of the left circumflex artery. Catheterization was done with a premedication consisting of $500 \mathrm{mg}$ IVMP $24 \mathrm{~h}$ before angioplasty, while prednisone dose was increased to $37.5 \mathrm{mg} /$ day. After PCI he started a cardiac rehabilitation program, prednisone was tapered down and discontinued in 6 weeks (Figure 1A).

Prompt restoration of euthyroidism in patient \#3 associated with an improvement in heart function, thus allowing the treatment of the aneurysm by a minimally invasive endovascular technique.

At the end of the follow-up, patient \#1 developed a permanent hypothyroidism requiring levothyroxine replacement therapy, 3 months after prednisone discontinuation. It is not possible to exclude that the iodine load due to PCI might have contributed to a faster restoration of euthyroidism and to the following hypothyroidism. The remaining three patients had a transient hypothyroidism followed by the restoration of euthyroidism.

\section{Discussion}

We show for the first time that combined oral and parenteral treatment with steroids is effective in AIT2 patients not responsive to oral prednisone administration. AIT is a severe consequence of amiodarone treatment which may cause recurrence of arrhythmias and heart failure. The management can be challenging and patients who develop a deterioration of left ventricular function may require
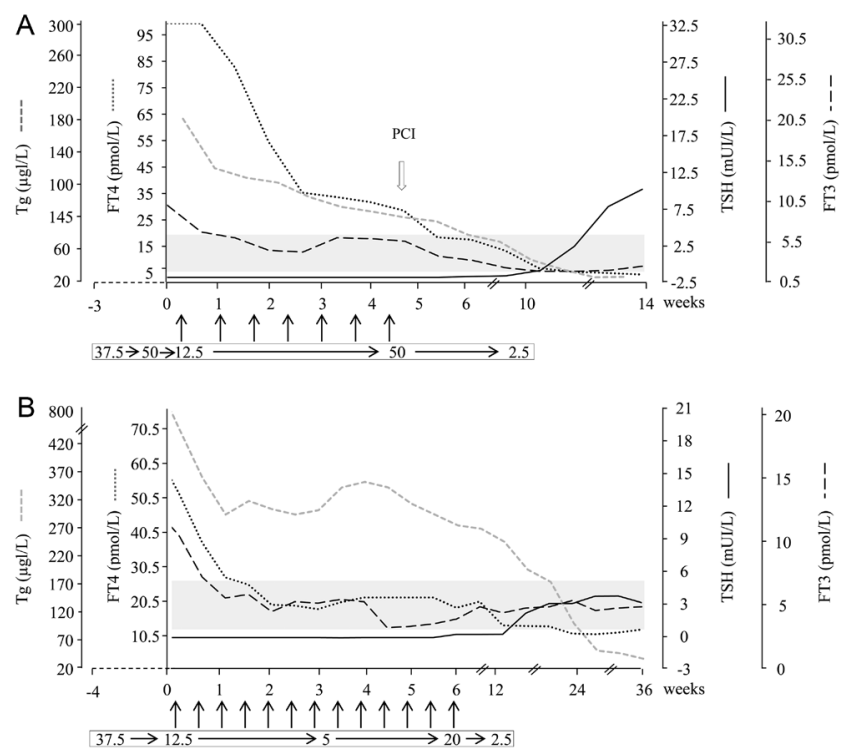

\section{Figure 1}

Trend of thyroid function tests and thyroglobulin during treatment in Patient 1 (panel A) and Patient 2 (panel B).

Reference ranges for TSH, FT4 and FT3 are highlighted with a gray box. Reference range: TSH 0.26-4.5 $\mu \mathrm{UI} / \mathrm{mL}$; FT3 2.9-

$7.1 \mathrm{pmol} / \mathrm{L} ; \mathrm{FT} 4$ 11.5-24.5 pmol/L; Tg 0-85 $\mu \mathrm{g} / \mathrm{L}$. The vertical black arrows indicate the IVMP pulses, while the white boxes the doses and timing of prednisone administration (mg/day). The white arrow in panel A represents the coronagraphy.

a prompt restoration of euthyroidism. Nevertheless, in nearly $20 \%$ of AIT2 cases, the time to restore euthyroidism with prednisone, which is the treatment of choice, is 90 days (range: 77-103 days) (6). In particular, a delayed response to glucocorticoids is observed when serum basal free T4 concentration are $>50 \mathrm{pg} / \mathrm{mL}$ ( $64 \mathrm{pmol} / \mathrm{L}$ ) and the thyroid volume is $\geq 12 \mathrm{~mL} / \mathrm{m}^{2}$ surface body area (6).

This time lapse is often unacceptable for patients with underlying severe cardiac disorders. Unfortunately, due to the destructive process underlying AIT2, radioiodine treatment can be performed only exceptionally in those patients who retain ${ }^{131} \mathrm{I}$ uptake, while urgent total thyroidectomy is often too risky in these compromised patients.

The combined treatment with prednisone and IVMP allowed the achievement of euthyroidism within 40 days in our four patients poorly responsive to prednisone administration. Although our patients had several comorbidities, only mild adverse events were observed.

The higher efficacy and the prompt response to IVMP with respect to prednisone is likely due to the higher doses 

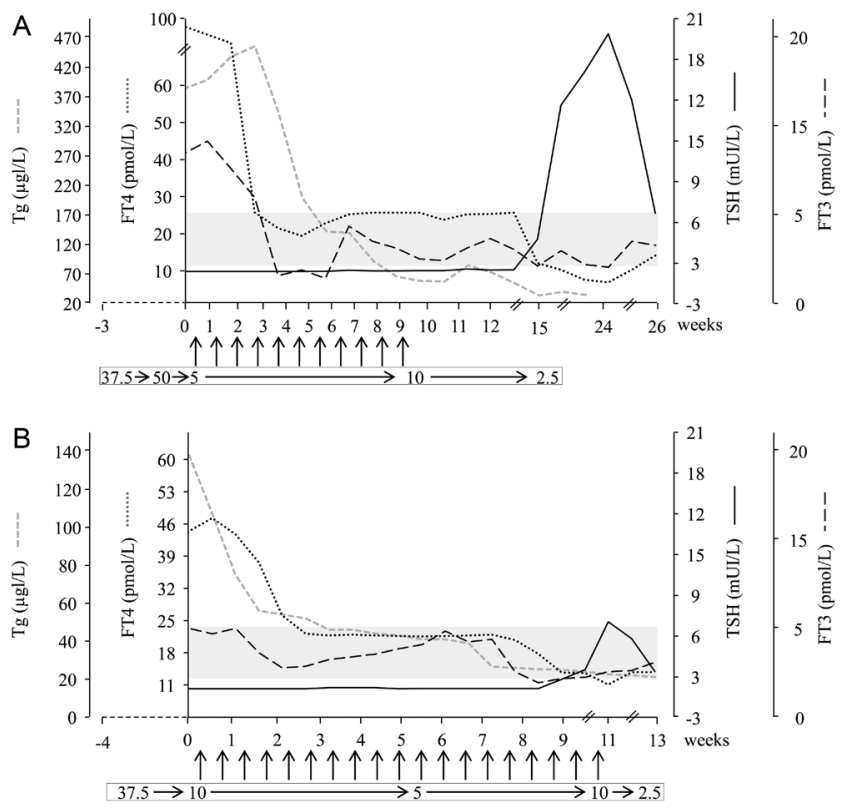

\section{Figure 2}

Trend of thyroid function tests and thyroglobulin during treatment in Patient 3 (panel A) and Patient 4 (panel B).

Reference range for TSH, FT4 and FT3 are highlighted with a gray box. Reference range: TSH 0.26-4.5 $\mu \mathrm{Ul} / \mathrm{mL}$; FT3 2.9-7.1 pmol/L; FT4 11.5-24.5 pmol/L; Tg 0-85 $\mu \mathrm{g} / \mathrm{L}$. The vertical black arrows indicate the IVMP pulses, while the white boxes the doses and timing of prednisone administration (mg/day).

which can be administered by the iv route, $500 \mathrm{mg}$ IVMP corresponding to $625 \mathrm{mg}$ of prednisone. Moreover, MP has a higher anti-inflammatory effect than prednisone (7). MP is often administrated intravenously in an intermittent fashion, in order to deliver higher corticosteroid doses and reduce long-term adverse events. Indeed, steroids can cause or worsen diabetes, psychosis, major depression, hypertension and weight gain, thus increasing the risk of adverse events in patients with cardiovascular diseases.

These effects are more common when steroid are given orally, while parenteral administration is more frequently associated with symptoms occurring during infusion or within $24 \mathrm{~h}$ of treatment (palpitations, flushes, and transient dyspepsia). Serious hepatic and cardiological adverse events occurred in patients receiving single doses higher than $0.5 \mathrm{~g}(8,9)$. In addition, a cumulative doses exceeding $8 \mathrm{~g}$ has been associated with increasing risk of severe liver damage $(10,11,12)$. Nevertheless, the treatment in a hospital setting with continuous ECG monitoring during IVMP and over the following 24 hours should be considered especially for patients with severe cardiomyopathies.
The limitations of this study are mainly related to the small number of patients included. However, the occurrence of an AIT2 not responsive to prednisone is an uncommon event as the majority of patients have a less severe form of hyperthyroidism and the thyroid function normalizes in 3-4 weeks. Moreover, we believe that the results obtained in this pilot study, though certainly requiring a confirmation in a randomized controlled study on a larger number of patients, could have a promising impact in clinical practice.

It is worth underlining that three out of four patients had an undiagnosed simple goiter, either measured as absolute volume or BSA-corrected thyroid volume, when amiodarone had been administered, highlighting that risk stratification, including at least US-thyroid scan and TSH measurement is advisable in patients older than 65 years undergoing long-term iodine load (amiodarone or even repeated administration of iodate contrast media). This may prevent or at least allow prompt treatment of thyrotoxicosis, which can be associated with increased morbidity and mortality. Still, prophylactic radioiodine ablation or total thyroidectomy may be reasonable in selected patients with goiter, requiring long-term amiodarone administration.

To the best of our knowledge, this is the first study exploring the efficacy of a combined parenteral and oral steroid therapy in the treatment of severe forms of AIT2. This schedule allowed us to deliver high steroid dose (corresponding to a daily prednisone dose between 110 and $223 \mathrm{mg}$ ) without significant side effects. With appropriate selection of patients and careful monitoring during and after treatment, this treatment is safe, well tolerated, and leads to a rapid improvement of thyroid function, avoiding urgent total thyroidectomy.

\section{Declaration of interest}

The authors declare that there is no conflict of interest that could be perceived as prejudicing the impartiality of the research reported.

\section{Funding}

This study was partially supported by the Italian Ministry of Education, University and Research: RC 02C502-2005.

\section{References}

1 Sarne D. Effects of the Environment, Chemicals and Drugs on Thyroid Function. South Dartmouth, MA: Endocrine Education Inc., 2016. (available at: http://www.thyroidmanager.org). Accessed on 30 January 2019.

2 Bogazzi F, Bartalena L \& Martino E. Approach to the patient with amiodarone-induced thyrotoxicosis. Journal of Clinical Endocrinology 
and Metabolism 201095 2529-2535. (https://doi.org/10.1210/ jc.2010-0180)

3 Bartalena L, Bogazzi F, Chiovato L, Hubalewska-Dydejczyk A, Links TP \& Vanderpump M. 2018 European Thyroid Association (ETA) guidelines for the management of amiodarone-associated thyroid dysfunction. European Thyroid Journal 20187 55-66. (https:// doi.org/10.1159/000486957)

4 Zhu L, Zainudin SB, Kaushik M, Khor LY \& Chng CL. Plasma exchange in the treatment of thyroid storm secondary to type II amiodarone-induced thyrotoxicosis. Endocrinology, Diabetes \& Metabolism Case Reports 20162016 160039. (https://doi.org/10.1530/ EDM-16-0039)

5 Mosteller RD. Simplified calculation of body-surface area. The New England Journal of Medicine 1987317 1098. (https://doi.org/10.1056/ NEJM198710223171717)

6 Bogazzi F, Bartalena L, Tomisti L, Rossi G, Tanda ML, Dell'Unto E, Aghini-Lombardi F \& Martino E. Glucocorticoid response in amiodarone-induced thyrotoxicosis resulting from destructive thyroiditis is predicted by thyroid volume and serum free thyroid hormone concentrations. Journal of Clinical Endocrinology and Metabolism 200792 556-562. (https://doi.org/10.1210/jc.2006-2059)

7 Schimmer BP \& Parker KL. Adrenocorticotropic hormones; adrenocortical steroids and their synthetic analogs; inhibitors of the synthesis and actions of adrenocortical hormones. In Goodman \& Gilman's the Pharmacological Basis of Therapeutics, 11th ed., pp 1587-1595, ch 59. Eds LL Brunton, JS Lazo \& KL Parker. New York: McGraw-Hill Professional, 2006.

8 Zang S, Ponto KA \& Kahaly GJ. Clinical review: intravenous glucocorticoids for Graves' orbitopathy: efficacy and morbidity. Journal of Clinical Endocrinology and Metabolism 201196 320-332. (https://doi.org/10.1210/jc.2010-1962)

9 Vasheghani-Farahani A, Sahraian MA, Darabi L, Aghsaie A \& Minagar A. Incidence of various cardiac arrhythmias and conduction disturbances due to high dose intravenous methylprednisolone in patients with multiple sclerosis. Journal of the Neurological Sciences 2011309 75-78. (https://doi.org/10.1016/j.jns.2011.07.018)

10 Le Moli R, Baldeschi L, Saeed P, Regensburg N, Mourits MP \& Wiersinga WM. Determinants of liver damage associated with intravenous methylprednisolone pulse therapy in Graves' ophthalmopathy. Thyroid 200717 357-362. (https://doi.org/10.1089/ thy.2006.0267)

11 Weissel M \& Hauff W. Fatal liver failure after high-dose glucocorticoid pulse therapy in a patient with severe thyroid eye disease. Thyroid 200010 521. (https://doi.org/10.1089/ thy.2000.10.521)

12 Marinó M, Morabito E, Brunetto MR, Bartalena L, Pinchera A $\&$ Marocci C. Acute and severe liver damage associated with intravenous glucocorticoid pulse therapy in patients with Graves' ophthalmopathy. Thyroid 200414 403-406. (https://doi. org/10.1089/105072504774193276)

Received 10 July 2019

Revised version received 26 August 2019

Accepted 17 September 2019 